\title{
MULTIVARIATE POLYNOMIAL INEQUALITIES VIA PLURIPOTENTIAL THEORY AND SUBANALYTIC GEOMETRY METHODS
}

\author{
WIESŁAW PLEŚNIAK \\ Instytut Matematyki, Uniwersytet Jagiellonski \\ Reymonta 4, 30-059 Kraków, Poland \\ E-mail: plesniak@im.uj.edu.pl
}

Dedicated to Professor Zbigniew Ciesielski in honour of his 70th birthday

\begin{abstract}
We give a state-of-the-art survey of investigations concerning multivariate polynomial inequalities. A satisfactory theory of such inequalities has been developed due to applications of both the Gabrielov-Hironaka-Łojasiewicz subanalytic geometry and pluripotential methods based on the complex Monge-Ampère operator. Such an approach permits one to study various inequalities for polynomials restricted not only to nice (nonpluripolar) compact subsets of $\mathbb{R}^{n}$ or $\mathbb{C}^{n}$ but also their versions for pieces of semialgebraic sets or other "small" subsets of $\mathbb{R}^{n}\left(\mathbb{C}^{n}\right)$.
\end{abstract}

I. Global polynomial inequalities. Multivariate polynomial inequalities are closely related to the Siciak extremal function associated with a compact subset $E$ of $\mathbb{C}^{n}$,

$$
\Phi_{E}(z)=\sup \left\{|p(z)|^{1 / \operatorname{deg} p}\right\}
$$

where $p: \mathbb{C}^{n} \rightarrow \mathbb{C}$ is a nonconstant polynomial with $\sup |p|(E) \leq 1, z \in \mathbb{C}^{n}$. Siciak's function establishes an important link between polynomial approximation in several variables and pluripotential theory. This yields its numerous applications in complex and real analysis. It is known (Zakharyuta 1976, Siciak 1981) that

$$
\log \Phi_{E}(z)=V_{E}(z):=\sup \left\{u(z): u \in \mathcal{L}\left(\mathbb{C}^{n}\right), u \leq 0 \text { on } E\right\},
$$

2000 Mathematics Subject Classification: 32B20, 14P05, 32E30, 32U35, 26E10, 41A17.

Key words and phrases: multivariate Bernstein-Walsh and Markov type inequalities, Siciak and Zakharyuta extremal functions, pluripotential theory, subanalytic geometry, approximation and extension of $C^{\infty}$ functions, algebraic sets, generalized Bernstein property.

Research partially supported by the KBN grant No 2 P03A 04722.

The paper is in final form and no version of it will be published elsewhere. 
where $\mathcal{L}\left(\mathbb{C}^{n}\right)=\left\{u \in P S H\left(\mathbb{C}^{n}\right): u(z)-\log |z| \leq O(1)\right.$ as $\left.|z| \rightarrow \infty\right\}$ is the Lelong class of plurisubharmonic functions with logarithmic growth at infinity. If $E$ is nonpluripolar (i.e. there is no plurisubharmonic function $u$ such that $E \subset\{u(z)=-\infty\}$ ), then the plurisubharmonic function

$$
V_{E}^{*}(z)=\limsup _{w \rightarrow z} V_{E}(w)
$$

is the unique function in the class $\mathcal{L}\left(\mathbb{C}^{n}\right)$ which vanishes on $E$ except perhaps for a pluripolar subset and satisfies the complex Monge-Ampère equation $\left(\operatorname{dd}^{c} V_{E}^{*}\right)^{n}=0$ in $\mathbb{C}^{n} \backslash E$ (Bedford and Taylor 1982). If $n=1$, the Monge-Ampère equation reduces to the classical Laplace equation. For this reason, the function $V_{E}^{*}$ is considered as a natural counterpart of the classical Green function with logarithmic pole at infinity and it is called the pluricomplex Green function associated with $E$.

By the definition of $\Phi_{E}$ one has

$$
|p(z)| \leq \sup |p|(E)\left[\Phi_{E}(z)\right]^{\operatorname{deg} p}, \quad z \in \mathbb{C}^{n},
$$

for each polynomial $p \in \mathbb{C}[z]$, which is a Bernstein-Walsh type inequality. Therefore the crucial point for applications is to establish the continuity of $\Phi_{E}$ in $\mathbb{C}^{n}$, which is equivalent, by Zakharyuta 1976 and Siciak 1983, to the following property

(B-W) For each $b>1$ there exists a neighbourhood $U$ of $E$ and a constant $M>0$ such that

$$
\sup |p|(U) \leq M b^{\operatorname{deg} p} \sup |p|(E) \quad \text { for each polynomial } p \in \mathbb{C}[z] .
$$

In such a case the set $E$ is said to be pluriregular or else $L$-regular.

Let us notice the following useful result:

Analytic AcCessibility Criterion (Sadullaev 1980, Pleśniak 1980, 1984a, 1984b, Cegrell 1985). Given $a \in E$, suppose there exists an analytic map $h:[0,1] \rightarrow E$ such that $h(0)=a$. If for each $t \in(0,1]$ the function $\Phi_{E}$ is continuous at $h(t)$ then $\Phi_{E}$ is also continuous at $a$.

$L$-regularity is invariant under nondegenerate holomorphic maps:

Theorem (Pleśniak 1978, Klimek 1981,1982, Sadullaev 1981, Nguyen Thanh Van and Pleśniak 1984). If $E$ is a compact, polynomially convex L-regular subset of $\mathbb{C}^{n}$ and $h: U \rightarrow \mathbb{C}^{m}(m \leq n)$, where $U$ is an open neighbourhood of $E$, is a nondegenerate holomorphic map (i.e. $\operatorname{rank}_{V} f=m$ for every connected component $V$ of $U$ such that $V \cap E \neq \emptyset)$, then the set $h(E)$ is also L-regular (in $\mathbb{C}^{m}$ ).

The analytic accessibility criterion via techniques of subanalytic geometry permits one to show that

THEOREM (Pleśniak 1984b). If $E$ is a compact set in $\mathbb{C}^{n}$ that is subanalytic as a subset of $\mathbb{R}^{2 n}$, and if int $E$ is dense in $E$ then $E$ is (locally) L-regular at every point $a \in E$.

This result is also valid for definable sets in some polynomially bounded o-minimal structures which are essential generalizations of subanalytic geometry (Pleśniak 2003, Pierzchała 2005). Let us recall that a subset $E$ of $\mathbb{R}^{n}$ is said to be semianalytic if for each 
point $x \in \mathbb{R}^{n}$ one can find a neighbourhood $U$ of $x$ and a finite number of real analytic functions $f_{i j}$ and $g_{i j}$ defined in $U$, such that

$$
E \cap U=\bigcup_{i} \bigcap_{j}\left\{f_{i j}>0, g_{i j}=0\right\}
$$

The projection of a semianalytic set need not be semianalytic (Łojasiewicz 1964). The class of sets obtained by enlarging that of semianalytic sets to include images under the projections has been called the class of subanalytic sets. More precisely, a subset $E$ of $\mathbb{R}^{n}$ is said to be subanalytic if for each point $x \in \mathbb{R}^{n}$ there exists an open neighbourhood $U$ of $x$ such that $E \cap U$ is the projection of a bounded semianalytic set $A$ in $\mathbb{R}^{n+m}$, where $m \geq 0$. If $n \geq 3$, the class of subanalytic sets is essentially larger than that of semianalytic sets, the classes being identical if $n \leq 2$.

The result about the $L$-regularity of subanalytic sets can be significantly strengthened with the aid of the Hironaka Rectilinearization Theorem and Eojasiewicz's Inequality, viz.

ThEOREM (Pawłucki and Pleśniak 1986). If $U$ is a non-void bounded open subanalytic set in $\mathbb{R}^{n}$ then the extremal function $\Phi_{E}$, with $E=\bar{U}$, has the following Hölder Continuity Property

$(H C P)$

$$
\Phi_{E}(z) \leq 1+A \delta^{m}, \quad \text { if } \operatorname{dist}(z, E) \leq \delta \leq 1,
$$

where the positive constants $A$ and $m$ depend only on the set $E$.

Actually, the last statement remains valid for an essentially larger family of uniformly polynomially cuspidal subsets of $\mathbb{R}^{n}$ (see Pawłucki and Pleśniak 1986).

Definition. A subset $E$ of $\mathbb{R}^{n}$ is said to be uniformly polynomially cuspidal (briefly, $U P C$ ) if one can choose constants $M>0, m \geq 1$ and $d \in \mathbb{N}$, and a mapping $h$ : $\bar{E} \times[0,1] \rightarrow \bar{E}$ such that for each $x \in \bar{E}, h(x, 1)=x, h(x, \cdot)$ is a polynomial map of degree $\leq d$ and

$$
\operatorname{dist}\left(h(x, t), \mathbb{R}^{n} \backslash E\right) \geq M(1-t)^{m}
$$

for $(x, t) \in \bar{E} \times[0,1]$.

Let us notice that some new examples of UPC sets have been recently found by Pierzchała (2005) in o-minimal structures generated by certain Denjoy-Carleman quasianalytic functions.

By the Cauchy Integral Formula, the (HCP) property of $\Phi_{E}$ yields the following Markov Inequality:

For any polynomial $P \in \mathbb{C}[z]$, one has

$$
\mid \text { gradient } P(z)\left|\leq M(\operatorname{deg} P)^{r} \sup \right| P \mid(E)
$$

for $z \in E$ with some positive constants $M$ and $r$ that do not depend on $P$.

Markov's Inequality, alongside the Bernstein-Walsh Inequality, is one of the fundamental tools of the Constructive Function Theory. Combined with Jackson's Theorem, it permits one to characterize $C^{\infty}$ functions on compact subsets of $\mathbb{R}^{n}$.

Theorem (Pawłucki and Pleśniak 1986, Pleśniak 1990). If a compact set $E$ in $\mathbb{R}^{n}$ is $C^{\infty}$ determining (i.e. for every $f \in C^{\infty}(E)$, if $f=0$ on $E$ then for each $\alpha \in \mathbb{N}_{0}^{n}, D^{\alpha} f=0$ on $E)$ then the following statements are equivalent: 
(i) E admits Markov's Inequality (MI);

(ii) (Bernstein's Theorem) For every function $f: E \rightarrow \mathbb{R}$, if the sequence $\left\{\right.$ dist $\left._{E}\left(f, \mathcal{P}_{k}\right)\right\}$ is rapidly decreasing (i.e. for each $s>0, k^{s} \operatorname{dist}_{E}\left(f, \mathcal{P}_{k}\right) \rightarrow 0$ as $k \rightarrow \infty$ ), then $f$ extends to a $C^{\infty}$ function $\tilde{f}$ in $\mathbb{R}^{n}$.

Let us also recall an important application of (MI) in differential analysis. This is a relatively simple construction of a continuous linear operator extending Whitney jets of $C^{\infty}$ functions from a compact subset of $\mathbb{R}^{n}$ that preserves (MI), to the whole space $\mathbb{R}^{n}$.

Theorem (Pawłucki and Pleśniak 1988). Let E be a Markov compact subset of $\mathbb{R}^{n}$. Then there exists a continuous linear operator

$$
L: \mathcal{C}^{\infty}(E) \rightarrow \mathcal{C}^{\infty}\left(\mathbb{R}^{n}\right)
$$

such that $L f_{\mid E}=f$ for each $f \in \mathcal{C}^{\infty}(E)$. Moreover, such an operator can be defined by

$$
L f=u_{1} L_{1} f+\sum_{k=1}^{\infty} u_{k}\left(L_{k+1} f-L_{k} f\right),
$$

where $L_{k} f$ is a Lagrange interpolation polynomial of $f$ of degree $k$ with nodes at FeketeLeja extremal points of $E$ and $u_{k}$ are appropriately chosen $C^{\infty}$ cut-off functions whose germs on $E$ are equal to 1.

Here $C^{\infty}(E):=\left\{f: E \rightarrow \mathbb{R} ; \exists g \in C^{\infty}\left(\mathbb{R}^{n}\right), g_{\mid E}=f\right\}$ is given the usual quotient topology of $C^{\infty}\left(\mathbb{R}^{n}\right) /\left\{f \in \mathbb{C}^{\infty}\left(\mathbb{R}^{n}\right) ; f=0\right.$ on $\left.E\right\}$.

(For another construction of an extension operator see Zeriahi 1993.) Actually, Markov's property of $E$ is equivalent to the existence of the extension operator $L$, if the space $C^{\infty}(E)$ is endowed with Jackson's topology defined by the seminorms $\|f\|_{E},\|f\|_{E}+$ $\sup _{k} k^{s} \operatorname{dist}\left(f, \mathcal{P}_{k}\right), s=1,2, \ldots$ (Pleśniak 1990). The open problem of whether the same holds true for $C^{\infty}(E)$ equipped with the standard topology was solved in the negative by Goncharov 1996, who constructed a non-Markov compact subset $E$ of $\mathbb{R}$ that admits the existence of a continuous linear operator extending $C^{\infty}$ functions from $E$ to the whole space $\mathbb{R}$.

For a Markov compact subset $E$ of $\mathbb{R}^{n}$, one can also construct a continuous linear operator extending jets of ultradifferentiable functions on $E$ to the whole space $\mathbb{R}^{n}$ with control of the loss of regularity (see Pleśniak and Skiba 1990, Pleśniak 1994, Beaugendre 2001).

Another natural domain yielding examples of $L$-regular and $H C P$-sets is complex dynamics. Given a polynomial mapping $P: \mathbb{C}^{n} \rightarrow \mathbb{C}^{n}$, we define the Eojasiewicz exponent of $P($ at $\infty)$ to be the number

$$
\nmid(P):=\sup \left\{\delta: \liminf _{|z| \rightarrow \infty} \frac{|P(z)|}{|z|^{\delta}}>0\right\} .
$$

The set $J_{P}$ given by the formula

$$
J_{P}:=\left\{z \in \mathbb{C}^{n}:\left\{P^{j}(z)\right\}_{j \in \mathbb{N}_{0}} \text { is bounded }\right\}
$$

is said to be the filled-in Julia set associated with the polynomial mapping $P$. Filled-in Julia sets in $\mathbb{C}^{n}$ arising from iterations of polynomial maps with Łojasiewicz's exponent 
strictly greater than 1 are $L$-regular (Klimek 1995, 1999). Actually, Klimek's sets have the (HCP) property (Kosek 1997, 1998).

II. Polynomial inequalities on lower dimensional sets. In recent years, Markov and Bernstein type inequalities have been intensively investigated on algebraic subvarieties of $\mathbb{R}^{n}$ (Fefferman and Narasimhan, Roytwarf and Yomdin, Bos, Levenberg, Milman and Taylor, Brudny̌, Baran and Pleśniak, Gendre, Kosek, Comte and Yomdin, Erdélyi and Kroó, Kroó and Szabados). The notion of Siciak's extremal function extends naturally to compact subsets of analytic sets in $\mathbb{C}^{n}$ (Sadullaev 1983, Zeriahi 1987, 1991, 1996, 2000). We have the following beautiful characterization of algebraicity of analytic subsets of $\mathbb{C}^{n}$. THEOREM (Sadullaev 1983). An analytic subset $A$ of $\mathbb{C}^{n}$ is algebraic if and only if the extremal function $\Phi_{E}$ is locally bounded on $A$ for some (and hence for each) nonpluripolar compact subset $E$ of $A$.

Sadullaev's criterion is crucial for the study of polynomial inequalities (of BernsteinWalsh, Markov or van der Corput-Schaake type) on algebraic sets (Baran and Pleśniak 2000a). It also plays a fundamental role in characterizing compact pieces of an algebraic variety in $\mathbb{C}^{n}$ in terms of tangential Markov, Bernstein or van der Corput-Schaake inequalities (Bos, Levenberg and Taylor 1995, Bos, Levenberg, Milman and Taylor 1995, Baran and Pleśniak 1997, 2000a, 2000b). Let us add that the techniques developed by Baran and Pleśniak 2000 are based on fine bounds for Siciak's extremal function associated with a ball in $\mathbb{R}^{n}$ which are due to Baran 1988, 1992, 1998. The tangential Markov inequality with exponent 1 characterizes the property of a compact subset $K$ of $\mathbb{R}^{N}$ to be a piece of an algebraic variety. More precisely, we have

THEOREM (Baran and Pleśniak 2000b). Let $K$ be a compact subset of $\mathbb{R}^{N}$ admitting an analytic parametrization $\phi$ of order $M(1 \leq M \leq N)$. Then the Zariski dimension of $K$ is $M$ iff there exists a constant $C>0$ such that

$$
\left|D_{T(t, v)} P(x)\right| \leq C(\operatorname{deg} P)\|P\|_{K}
$$

for $x \in K$ and for every polynomial $P \in \mathbb{C}\left[x_{1}, \ldots, x_{N}\right]$, where $T(t, v)=D_{v} \phi(t)$, the derivative of $\phi$ in direction $v$.

If $K$ is a smooth compact subvariety of $\mathbb{R}^{N}$ of dimension $M, 1 \leq M \leq N$, the above theorem was earlier proved by Bos, Levenberg, Milman and Taylor 1995.

If $E$ is a polynomially convex, HCP compact subset of $\mathbb{C}^{M}$ with HCP-exponent $m$ and $f$ is a nondegenerate analytic map defined in an open neighborhood of $E$, with values in an algebraic subset $\mathbb{M}$ of $\mathbb{C}^{N}$ of dimension $M, 1 \leq M \leq N$, then we have

Theorem (Baran and Pleśniak 2000a). There exists a constant $C>0$ such that for every polynomial $Q \in \mathbb{C}\left[z_{1}, \ldots, z_{N}\right]$ we have

$$
\left|D_{T(t, v)} Q(f(t))\right| \leq C_{1} d^{1 / m}\|Q\|_{f(E)},
$$

where $t \in E$ and $T(t, v)=D_{v} f(t)$, the derivative of $f$ in direction $v$.

COROLlaRY. Let $f$ be a polynomial map from $\mathbb{R}$ to $\mathbb{R}^{n}$ with

$$
f^{\prime}(t)=(1-t)^{s_{1}}(1+t)^{s_{2}} Q(t),
$$


where $Q$ is a polynomial map from $\mathbb{R}$ to $\mathbb{R}^{n}, Q(t) \neq 0$ on $[-1,1]$. Let $\alpha=\max \left(s_{1}, s_{2}\right)$. Then there exists a constant $A>0$ such that for any polynomial $P \in \mathbb{R}\left[x_{1}, \ldots, x_{n}\right]$ of degree $d$ we have

$$
\left|D_{\mathcal{T}_{x}} P(x)\right| \leq A d^{2+2 \alpha}\|P\|_{N}, \text { for } x \in N=f([-1,1])
$$

with $D_{\mathcal{T}_{x}} P(x)=D_{(Q(t) /\|Q(t)\|)} P(x), x=f(t)$.

EXAMPLE. Let $m$ and $l$, where $m>l \geq 2$, be two relatively prime natural numbers. Let $f(t)=\left(\left(\frac{1+t}{2}\right)^{l},\left(\frac{1+t}{2}\right)^{m}\right)$. Then

$$
N=f([-1,1])=\left\{(x, y) \in \mathbb{R}^{2}: 0 \leq x, y \leq 1 \text { and } x^{m}=y^{l}\right\} .
$$

Since in this case $\alpha=l-1$, by the above corollary we get

$$
\left|D_{\mathcal{T}_{(x, y)}} P(x, y)\right| \leq A d^{2 l}\|P\|_{N},
$$

for any polynomial $P \in \mathbb{R}[x, y]$ of degree $d$. (See also Bos-Levenberg-Milman-Taylor 1998, Gendre 1998.)

On the other hand, Bos, Levenberg, Milman and Taylor (1995) gave an example of an analytic function $f:[0,1] \rightarrow \mathbb{R}$ such that $f([0,1])$ does not admit a tangential Markov inequality with any finite exponent. Therefore one could conjecture that such an inequality holds iff $f([0,1])$ is a piece of an algebraic variety. This was disproved by a recent result of Bos, Brudnyı̆, Levenberg and Totik 2003:

THEOREM. Given a polynomial $T \in \mathbb{R}[x]$, for any interval $[a, b] \subset \mathbb{R}$, there exists a constant $C>0$ such that for any polynomial $P \in \mathbb{C}[x, y]$ we have

$$
\max _{x \in[a, b]}\left|\frac{d}{d x} P\left(x, e^{T(x)}\right)\right| \leq C(\operatorname{deg} P)^{4} \max _{x \in[a, b]}\left|P\left(x, e^{T(x)}\right)\right| .
$$

Moreover, the exponent 4 is optimal.

III. A Generalized Bernstein Inequality. Let $K$ be a non-pluripolar compact subset of $\mathbb{C}^{N}$ and let $V_{1} \subset V_{2} \subset \cdots \subset \mathcal{O}(\Omega)$ be a nested sequence of linear subspaces of the space $\mathcal{O}(\Omega)$ of holomorphic functions on an open bounded neighborhood $\Omega$ of the polynomial hull $\hat{K}$ of $K$. Let $\{\phi(n)\}_{n=1}^{\infty} \subset(0, \infty)$ be an increasing sequence tending to $\infty$.

Definition. The compact $K$ is said to have property (B) (of Bernstein) with respect to $\left\{V_{n}\right\}$ and $\{\phi(n)\}$ if there exists a positive constant $C=C(\Omega, K)$ such that for each $n \in \mathbb{N}$ and each $f \in V_{n}$, one has

$$
\sup _{\Omega}|f| \leq C^{\phi(n)} \sup _{K}|f| .
$$

\section{EXAMPLES.}

$1^{o}$ (Siciak 1962) Let $K$ be a non-pluripolar compact subset of $\mathbb{C}^{N}$ and let $V_{n}=$ $\mathbb{C}_{n}\left[z_{1}, \ldots, z_{N}\right]$, the space of algebraic polynomials of degree at most $d$ in $N$ complex variables $z_{1}, \ldots, z_{N}$. Then the Siciak extremal function

$$
\Phi_{K}(z)=\sup \left\{|P(z)|^{1 / \operatorname{deg} P} ; P \in \mathbb{C}[z], \sup _{K}|P| \leq 1\right\}
$$


is locally bounded in $\mathbb{C}^{N}$. Hence for any bounded open neighborhood $\Omega$ of $K$ we have

$$
\sup _{\Omega}|P| \leq C^{n} \sup _{K}|P|, \quad \text { with } C=\sup _{\Omega} \Phi_{K} .
$$

$2^{o}$ (Baouendi 1974) Let $D$ be a bounded open set in $\mathbb{R}^{N}$ with Lipschitz boundary and let $A$ be a differential operator of order 2 with coefficients in the space $\mathcal{O}(\bar{D})$ of holomorphic functions in a neighbourhood of $\bar{D}$. We assume that $A$ is formally self-adjoint. We denote by $(A, \mathcal{D}(A))$ a strictly positive self-adjoint realization of $A$ in $L^{2}(D)$. We assume moreover that the embedding of $\mathcal{D}(A)$ into $L^{2}(D)$ is compact and that $A$ has the following iteration property: If $u \in \mathcal{D}\left(A^{\infty}\right)$ and if there exists a constant $L>0$ such that

$$
\left\|A^{k} u\right\|_{L^{2}(D)} \leq L^{k+1}(2 k) !
$$

for every $k \in \mathbb{N}$, then $u \in \mathcal{O}(\bar{D})$. (Such operators are generalizations of the classical Legendre operator $A=-\frac{d}{d x}\left(1-x^{2}\right) \frac{d}{d x}+1$ with $D=(-1,1)$.) We denote by $\left(\lambda_{j}\right)_{j \in \mathbb{N}}$ the nondecreasing sequence of eigenvalues of $A$ repeated according to their multiplicities, and by $\left(h_{j}\right)$ the sequence of eigenfunctions of $A$ associated to $\left(\lambda_{j}\right)$. We set $V_{n}=\operatorname{Lin}\left\{h_{0}, h_{1}, \ldots, h_{n}\right\}$. Then for every $b>1$ there exists a neighborhood $\Omega$ of $K$ in $\mathbb{C}^{N}$ such that for each $n \in \mathbb{N}$ and for each $f \in V_{n}, f$ extends holomorphically to $\Omega$ and

$$
\sup _{\Omega}|f| \leq b^{\sqrt{\lambda_{n}}} \sup _{K}|f| \text {. }
$$

$3^{\circ}$ (Tijdeman 1971) Let $K=\{z \in \mathbb{C}:|z| \leq 1\}$ and let $V_{n}=\left\{f(z)=P\left(z, e^{z}\right)\right): P \in$ $\left.\mathbb{C}_{n}[z, w]\right\}$, where $\mathbb{C}_{n}[z, w]$ denotes the space of all polynomials in $\mathbb{C}^{2}$ of degree at most $n$. For $R>1$, set $\Omega_{R}=\{z \in \mathbb{C}:|z|<R\}$. Then for $z \in \Omega_{R}$, for each $f \in V_{n}$ and for each $n \in \mathbb{N}$,

$$
|f(z)| \leq\|f\|_{K} e^{n^{2} \log R+6 n R} .
$$

$4^{o}$ (Coman and Poletsky 2003) Let $K=\left\{(z, f(z)) \in \mathbb{C}^{2}:|z| \leq 1\right\}$, where $f(z)=e^{z}$. Put $V_{n}=\mathbb{C}_{n}[z, w]$. Then there exists a constant $C>0$ such that for every $R>1$ and for every $P \in V_{n}(n=1,2, \ldots)$

$$
|P(z, w)| \leq\|P\|_{K} \exp \left(\frac{n^{2} \log n}{2}+C_{1} n^{2}+n \log R\right)
$$

for any $(z, w) \in \Omega_{R}:=\{\max \{|z|,|w|\}<R\}$.

$5^{\circ}$ (Bos, Brudnyı̆, Levenberg and Totik 2003) Let $T(x)$ be any fixed polynomial in $x \in \mathbb{R}$. Put $V_{n}=\left\{f(x)=P\left(x, e^{T(x)}\right): P \in \mathbb{C}_{n}[x, y]\right\}$ and $\phi(n)=n^{2}$. Then, if $0<R_{1}<R_{2}$, there exists a constant $C=C\left(R_{1}, R_{2}\right)$ such that for each $n \in \mathbb{N}$ and each $f \in V_{n}$,

$$
|f(z)| \leq C^{\phi(n)} \sup _{\left[-R_{1}, R_{2}\right]}|f|
$$

for each $z \in \mathbb{C}$ with $|z| \leq R_{2}$.

By a standard pluripotential theory argument, property (B) implies a Markov type inequality on a sufficiently regular compact set $K$. More precisely, we have

THEOREM. Suppose $V_{K}:=\log \Phi_{K}$ is Hölder continuous on $K$, i.e. there exist constants $M>0$ and $s \geq 1$ such that

$$
V_{K}(z) \leq M(\operatorname{dist}(z, K))^{1 / s} \quad \text { for } z \in \mathbb{C}^{N} .
$$


If the couple $(K, \Omega)$ has property $(\mathbf{B})$ with respect to $\left\{V_{n}\right\}$ and $\phi(n)$, then there exists a constant $C>0$ such that for each $n \in \mathbb{N}$ and for each $f \in V_{n}$ one has

$$
|\operatorname{grad} f(x)| \leq C(\phi(n))^{s}\|f\|_{K} \quad \text { for } x \in K \text {. }
$$

In the definition of property (B) no assumption on $\operatorname{dim} V_{n}$ is imposed. However, we have

THEOREM. If $K$ satisfies (B) with respect to $\left\{V_{n}\right\}$ and $\{\phi(n)\}$, then

$$
\operatorname{dim} V_{n}=O\left(\phi^{N}(n)\right) \text {. }
$$

The above theorem follows by combining the next two lemmas.

Uniform Bernstein-Walsh-Siciak Theorem (Pleśniak 1972). Let $\mathcal{H}^{\infty}(\Omega)$ be the space of all bounded holomorphic functions in an open set $\Omega \subset \mathbb{C}^{N}$. For every polynomially convex compact subset $K$ of $\Omega$ there exist constants $M>0$ and $a \in(0,1)$ such that for every $f \in \mathcal{H}^{\infty}(\Omega)$,

$$
\operatorname{dist}_{K}\left(f, \mathbb{C}_{n}[z]\right) \leq M \sup _{\Omega}|f| a^{n} .
$$

Krein-Krasnoselsky-Milman Lemma (cf. Singer 1970). Let $E$ be a normed linear space and $G_{1}, G_{2}$ two linear subspaces of $E$ such that

$$
\operatorname{dim} G_{1}<\infty, \quad \operatorname{dim} G_{1}<\operatorname{dim} G_{2} .
$$

Then there exists an element $y \in G_{2} \backslash\{0\}$ such that

$$
\|y+g\| \geq\|y\| \quad \text { for every } g \in G_{1} .
$$

It is well-known that

$$
V_{[a, b]}(z) \leq M(\operatorname{dist}(z,[a, b]))^{1 / 2}, \quad z \in \mathbb{C} .
$$

Hence by the first theorem of this section, the Bos-Brudnyı̆-Levenberg-Totik theorem with exponent 4 follows from property $(\mathrm{B})$ with $\phi(n)=n^{2}$.

In this case, the curve $\Gamma:=\left\{y=e^{T(x)}, x \in[a, b]\right\}$ is not algebraic (if $\left.T(x) \not \equiv 0\right)$, whence

$$
\operatorname{dim} V_{n}=\operatorname{dim}\left\{P\left(x, e^{T(x)}\right): P \in \mathbb{C}_{n}[x, y]\right\}=\operatorname{dim} \mathbb{C}_{n}[x, y]=\frac{n(n+1)}{2} .
$$

Therefore by our previous Theorem we must have

$$
\phi(n) \geq C n^{2}, \quad n=1,2, \ldots,
$$

independently of the choice of the polynomial $T$. Consequently, we get

COROLlaRY. The Bernstein exponent $\phi(n)=n^{2}$ of Bos-Brudny̆̌Levenberg-Totik's example $5^{\circ}$ is optimal.

\section{References}

M. S. Baouendi, 1974, Iterates of elliptic operators and Bernstein inequalities, in: Functional Analysis (Proc. of the Brazilian Mathematical Society Symposium), Sao Paulo, D. de Figueiredo (ed.), Lecture Notes in Pure and Applied Mathematics 18, Marcel Dekker, New York. 
M. Baran, 1988, Siciak's extremal function of convex sets in $\mathbb{C}^{n}$, Ann. Polon. Math. 48, 275-280.

M. Baran, 1992, Plurisubharmonic extremal function and complex foliations for the complement of convex sets in $\mathbb{R}^{n}$, Michigan Math. J. 39, 395-404.

M. Baran, 1998, Conjugate norms in $\mathbb{C}^{n}$ and related geometrical problems, Dissertationes Math. 377.

M. Baran and W. Pleśniak, 1997, Bernstein and van der Corput-Schaake type inequalities on semialgebraic curves, Studia Math. 125, 83-96.

M. Baran and W. Pleśniak, 2000a, Polynomial inequalities on algebraic sets, Studia Math. 141, 209-219.

M. Baran and W. Pleśniak, 2000b, Characterization of compact subsets of algebraic varieties in terms of Bernstein type inequalities, Studia Math. 141, 221-234.

P. Beaugendre, 2001, Extensions de jets dans des intersections de classes non quasi-analytiques, Ann. Polon. Math. 76, 213-243.

E. Bedford and B. A. Taylor, 1982, A new capacity for plurisubharmonic functions, Acta Math. $149,1-40$.

L. Bos, A. Brudnyı̆, N. Levenberg and V. Totik, 2003, Tangential Markov inequalities on transcendental curves, Constr. Approx. 19, 339-354.

L. Bos, N. Levenberg, P. Milman and B. A. Taylor, 1995, Tangential Markov inequalities characterize algebraic submanifolds of $\mathbb{R}^{N}$, Indiana Univ. Math. J. 44, 115-138.

L. Bos, N. Levenberg, P. Milman and B. A. Taylor, 1998, Tangential Markov inequalities on real algebraic varieties, Indiana Univ. Math. J. 47, 1257-1271.

L. Bos, N. Levenberg and B. A. Taylor, 1995, Characterization of smooth, compact algebraic curves in $\mathbb{R}^{2}$, in: Topics in Complex Analysis, P. Jakóbczak and W. Pleśniak (eds.), Banach Center Publ. 31, Inst. Math., Polish Acad. Sci. Warszawa, 125-134.

L. Bos and P. Milman, 1995, Sobolev-Gagliardo-Nirenberg and Markov type inequalities on subanalytic domains, Geometric and Functional Analysis 5, 853-923.

U. Cegrell, 1985, Thin sets in $\mathbb{C}^{n}$, Univ. Iagellon. Acta Math. 25, 115-120.

D. Coman and E. A. Poletsky, 2003, Bernstein-Walsh inequalities and the exponential curve in $\mathbb{C}^{2}$, Proc. Amer. Math. Soc. 131, 879-887.

L. Gendre, 1998, Inégalité de Markov tangentielle locale sur les courbes algébriques de $\mathbb{R}^{n}$, Université Paul Sabatier, Toulouse (preprint).

A. Goncharov, 1996, A compact set without Markov's property but with an extension operator for $\mathcal{C}^{\infty}$ functions, Studia Math. 119, 27-35.

M. Klimek, 1981, A note on the L-regularity of compact sets in $\mathbb{C}^{n}$, Bull. Acad. Polon. Sci. Série Sci. Math. 29, 449-451.

M. Klimek, 1982, Extremal plurisubharmonic functions and L-regular sets in $\mathbb{C}^{n}$, Proc. Roy. Irish Acad. 82A, 217-230.

M. Klimek, 1995, Metrics associated with extremal plurisubharmonic functions, Proc. Amer. Math. Soc. 123, 2763-2770.

M. Klimek, 1999, Inverse iteration systems in $\mathbb{C}^{n}$, Acta Univ. Uppsaliensis 64, 206-214.

M. Kosek, 1997, Hölder continuity property of filled-in Julia sets in $\mathbb{C}^{n}$, Proc. Amer. Math. Soc. 125, 2029-2032.

M. Kosek, 1998, Hölder continuity property of composite Julia sets, Bull. Polish Acad. Sci. Math. $46,391-399$.

M. Kosek, 2000, Iteration of polynomial mappings on algebraic sets, Complex Variables Theory Appl. 43, 187-197.

S. Łojasiewicz, 1964, Ensembles semi-analytiques, Inst. Hautes Etudes Sci., Bures-sur-Yvette. 
T.V. Nguyen and W. Pleśniak, 1984, Invariance of L-regularity and Leja's polynomial condition under holomorphic mappings, Proc. Roy. Irish Acad. 84A, 111-115.

W. Pawłucki and W. Pleśniak, 1986, Markov's inequality and $\mathcal{C}^{\infty}$ functions on sets with polynomial cusps, Math. Ann. 275, 467-480.

W. Pawłucki and W. Pleśniak, 1988, Extension of $\mathcal{C}^{\infty}$ functions from sets with polynomial cusps, Studia Math. 88, 279-287.

R. Pierzchała, 2005, UPC condition in polynomially bounded o-minimal structures, J. Approx. Theory $132,25-33$.

W. Pleśniak, 1972, On superposition of quasianalytic functions, Ann. Polon. Math. 26, 73-84.

W. Pleśniak, 1978, Invariance of the L-regularity of compact sets in $\mathbb{C}^{n}$ under holomorphic mappings, Trans. Amer. Math. Soc. 246, 373-383.

W. Pleśniak, 1980, Sur la L-régularité des compacts de $\mathbb{C}^{N}$, Polycopié de la Faculté des Sciences de Toulouse, Université Paul Sabatier, U.E.R. de Mathématiques - Informatique - Gestion, Toulouse, 46 pp.

W. Pleśniak, 1984a, A criterion for polynomial conditions of Leja's type in $\mathbb{C}^{N}$, Univ. Iagell. Acta Math. 24, 139-142.

W. Pleśniak, 1984b, L-regularity of subanalytic sets in $\mathbb{R}^{n}$, Bull. Polish Acad. Sci. Math. 32, 647-651.

W. Pleśniak, 1990, Markov's inequality and the existence of an extension operator for $\mathcal{C}^{\infty}$ functions, J. Approx. Theory 61, 106-117.

W. Pleśniak, 1994, Extension and polynomial approximation of ultradifferentiable functions in $\mathbb{R}^{n}$, Bull. Soc. Roy. Sci. Liège 63, 393-402.

W. Pleśniak, 2003, Pluriregularity in polynomially bounded o-minimal structures, Univ. Iagel. Acta Mathematica 41, 205-214.

W. Pleśniak, 2004, An estimate from below of a generalized Bernstein exponent, Institute of Mathematics, Jagiellonian University, Cracow (preprint).

W. Pleśniak and A. Skiba, 1990, Polynomial approximation and linear extension of Gevrey classes of functions on compact sets, Publ. IRMA, Lille 21(IV), 2-11.

A. Sadullaev, 1980, P-regularity of sets in $\mathbb{C}^{n}$, in: Lecture Notes in Math. 798, Springer-Verlag, $402-411$.

A. Sadullaev, 1981, Plurisubharmonic measures and capacities on complex manifolds, Russian Math. Surveys 365:4, 61-119.

A. Sadullaev, 1983, An estimate for polynomials on analytic sets, Math. USSR Izv. 20, 493-502.

J. Siciak, 1962, On some extremal functions and their applications in the theory of analytic functions of several complex variables, Trans. Amer. Math. Soc. 105, 322-357.

J. Siciak, 1981, Extremal plurisubharmonic functions in $\mathbb{C}^{n}$, Ann. Pol. Math. 39, 175-211.

I. Singer, 1970, Best Approximation in Normed Linear Spaces by Elements of Linear Subspaces, Springer-Verlag, Berlin-Heidelberg-New York.

R. Tijdeman, 1971, On the number of zeros of general exponential polynomials, Indag. Math. 37, $1-7$.

V. P. Zakharyuta, 1976, Extremal plurisubharmonic functions, orthogonal polynomials and Bernstein-Walsh theorem for analytic functions of several variables, Ann. Polon. Math. 33, 137138 (Russian).

A. Zeriahi, 1987, Meilleure approximation polynomiale et croissance des fonctions entières sur certaines variétés affines, Ann. Inst. Fourier (Grenoble) 37, 79-104.

A. Zeriahi, 1991, Fonction de Green pluricomplexe à pôle à l'infini sur un espace de Stein parabolique et applications, Math. Scand. 69, 89-126. 
A. Zeriahi, 1993, Inégalités de Markov et développement en série de polynômes orthogonaux des fonctions $\mathcal{C}^{\infty}$ et $\mathcal{A}^{\infty}$, in: Proceedings of the Special Year of Complex Analysis of the Mittag-Leffler Institute 1987-88, J. F. Fornaess (ed.), Princeton Univ. Press, Princeton New Jersey, 1993, 693-701.

A. Zeriahi, 1996, Pluricomplex Green functions and approximation of holomorphic functions, in: Complex Analysis, Harmonic Analysis and Applications (Bordeaux, 1995), Pitman Res. Notes Math. Ser. 347, 104-142.

A. Zeriahi, 2000, A criterion of algebraicity for Lelong classes and analytic sets, Acta Math. 184, $113-143$. 